\title{
SHEAR-DRIVEN INSTABILITIES IN HALL-MAGNETOHYDRODYNAMIC PLASMAS
}

\author{
Cecilia Bejarano $^{1}$, Daniel O. Gómez ${ }^{1,3}$, and Axel Brandenburg ${ }^{2,4}$ \\ ${ }^{1}$ Instituto de Astronomía y Física del Espacio (Consejo Nacional de Investigaciones Científicas y Técnicas, Universidad de Buenos Aires), \\ Ciudad Universitaria, 1428 C.A.B.A., Buenos Aires, Argentina; cbejarano@iafe.uba.ar, gomez@iafe.uba.ar \\ 2 NORDITA, AlbaNova University Center, Roslagstullsbacken 23, SE-10691 Stockholm, Sweden; brandenb@ nordita.org \\ Received 2010 December 21; accepted 2011 May 26; published 2011 August 4
}

\begin{abstract}
The large-scale dynamics of plasmas is well described within the framework of magnetohydrodynamics (MHD). However, whenever the ion density of the plasma becomes sufficiently low, the Hall effect is likely to become important. The role of the Hall effect has been studied in several astrophysical plasma processes, such as magnetic reconnection, magnetic dynamo, MHD turbulence, or MHD instabilities. In particular, the development of smallscale instabilities is essential to understand the transport properties in a number of astrophysical plasmas. The magneto-rotational instability (MRI), which takes place in differentially rotating accretion disks embedded in relatively weak magnetic fields, is just one example. The influence of the large-scale velocity flows on small-scale instabilities is often approximated by a linear shear flow. In this paper, we quantitatively study the role of the Hall effect on plasmas embedded in large-scale shear flows. More precisely, we show that an instability develops when the Hall effect is present, which we therefore term as the Hall magneto-shear instability. As a particular case, we recover the so-called MRI and quantitatively assess the role of the Hall effect on its development and evolution.
\end{abstract}

Key words: instabilities - magnetohydrodynamics (MHD) - plasmas

\section{INTRODUCTION}

The large-scale dynamics of astrophysical plasmas is described theoretically within the framework of magnetohydrodynamics (MHD). In many cases of interest, these astrophysical flows are characterized by extremely large Reynolds numbers, which in turn imply that a wide range of spatial scales are relevant to properly describe their dynamical behavior. At sufficiently small spatial scales, kinetic plasma processes might become non-negligible under certain circumstances. For instance, in a fully ionized plasma, whenever one reaches spatial scales as small as the ion skin depth $c / \omega_{\mathrm{pi}}$ (where $c$ is the speed of light and $\omega_{\mathrm{pi}}$ is the ion plasma frequency), the Hall effect should not be neglected. This regime corresponds to fully ionized plasmas with sufficiently low ion densities. However, it can also arise in cold plasmas with a low ionization fraction $\chi$ in which case the relevant Hall scale is given by $\left(c / \omega_{\mathrm{pi}}\right) \chi^{-1 / 2}$ (see Pandey \& Wardle 2008 for details on the Hall-MHD of partially ionized plasmas). In cold plasmas such as those present in protoplanetary disks, another kinetic effect known as ambipolar diffusion might become relevant (Brandenburg \& Zweibel 1995), especially toward the disk surface (Pandey \& Wardle 2008). The relative importance of non-ideal effects such as Hall, ohmic dissipation, and ambipolar diffusion has been extensively discussed by Balbus \& Terquem (2001) as well as by Pandey \& Wardle (2008). To highlight the relevance of the Hall effect in astrophysical plasmas, it is useful to compare the orders of magnitude of the ohmic $(O)$, inductive $(I)$, and Hall $(H)$ terms in the generalized Ohms's law. For example, in a typical protostellar disk, the relevant ratios are $H / O \sim 10^{2}$ and $H / I \sim 10^{4}$ (Balbus \& Terquem 2001); while, for dwarf nova disk $H / I \sim 1$ (Sano $\&$ Stone 2002a) and for the crust of a neutron star $H / O \sim 10^{3}$ (Hollerbach \& Rüdiger 2002).

\footnotetext{
3 Also at Departamento de Física, Facultad de Ciencias Exactas y Naturales, Universidad de Buenos Aires, Ciudad Universitaria, 1428 C.A.B.A., Buenos Aires, Argentina.

4 Also at Department of Astronomy, Stockholm University, SE-10691 Stockholm, Sweden.
}

There are many examples of astrophysical plasma flows for which the role of the Hall effect has been studied: regions of star formation (Norman \& Heyvaerts 1985), dense molecular clouds (Wardle \& Ng 1999), the interstellar medium (Spangler 1999; Kinney et al. 2000), or even the early universe (Tajima et al. 1992). Due to their intense magnetic fields, the Hall currents can also be relevant in white dwarfs and neutron stars (Urpin \& Yakovlev 1980; Shalybkov \& Urpin 1997; Potekhin 1999). Also, the role of Hall currents in the generation of magnetic fields by turbulent dynamo activity has been studied by Mininni et al. (2002); see also Mininni et al. (2003a, 2003b) and references therein.

Even though the dynamics of small-scale structures is often unobservable in astrophysical flows, they may play an important role through nonlinear interactions with the large-scale part of the flow. In many cases, the small-scale dynamics of the fluids is instrumental in changing the transport properties of the large-scale dynamics of the fluids, and therefore it is relevant to identify potential instabilities in the microscale. At these small spatial scales, the large-scale velocity field can be reasonably approximated by a linear shear flow. The so-called shear-driven instabilities are those that originate as a result of the presence of a large-scale velocity shear.

In this paper, we study the potential relevance of the Hall effect in the presence of an external magnetic field as well as a linear shear flow. In particular, we focus our attention on the following two types of flows with the Hall effect: (1) non-rotating shear flows leading to what we call Hall magnetoshear instability (Hall-MSI) and (2) differentially rotating flows leading to Hall magneto-rotational instability (or Hall-MRI). In the absence of both rotation and shear, the linear modes in HallMHD correspond to right-hand polarized whistlers and lefthand polarized ion-cyclotron waves (see, for instance, Mahajan et al. 2005 and references therein). When these modes propagate embedded in a shear flow, the ion-cyclotron mode might become unstable. This instability takes place when the shear is steep enough to be larger than the ion-cyclotron frequency. A linear analysis of this instability has been recently reported by Kunz 
(2008), for the case of weakly ionized plasmas. Another study on the influence of the Hall effect on weakly ionized plasmas subjected to differential rotation was reported by Rüdiger \& Kitchatinov (2005) for finite magnetic Reynolds numbers up to 300 (see also Rüdiger \& Shalybkov 2004).

In the present study, we adopt a one-dimensional configuration, perform a linear analysis to identify potential instabilities, and then compare with numerical simulations. The set of equations as well as the simplifying assumptions that we adopt are listed in Section 2. The dispersion relation for the linear regime is shown in Section 3. We briefly describe the numerical code employed in Section 4. The role of the Hall effect on non-rotating shear flows is presented in Section 5, while the action of Hall currents on the well-known MRI is discussed in Section 6. The nonlinear behavior is tackled through a qualitative approach in Section 7. Finally, we summarize our conclusions in Section 8.

\section{GENERAL EQUATIONS}

The dimensionless Hall-MHD equations in a rotating reference frame with angular velocity $\boldsymbol{\Omega}=\Omega_{0} \hat{\mathbf{z}}$ are listed below. We use $t_{0}$ as our time unit, velocities are in units of the Alfvén velocity $v_{A}$, and particle densities are in units of $n_{0}$. For a fully ionized hydrogen plasma, the continuity equation is

$$
\frac{\partial n}{\partial t}+\nabla \cdot(n \mathbf{u})=0
$$

where $n$ is both the proton and the electron particle density (i.e., $n_{e}=n_{i}=n$ ) to guarantee charge neutrality. The equation of motion for this plasma is

$$
\frac{\partial \mathbf{u}}{\partial t}+(\mathbf{u} \cdot \nabla) \mathbf{u}=-2 \beta \nabla h+\frac{\nabla \times \mathbf{B} \times \mathbf{B}}{n}-2 \Omega \hat{\mathbf{z}} \times \mathbf{u}+v \nabla^{2} \mathbf{u},
$$

where $\beta=\left(c_{s} / v_{A}\right)^{2}$ is the plasma parameter $\left(c_{s}\right.$ being the sound speed), $h$ is the enthalpy density for a barotropic flow for each species (electrons or protons), $\Omega=\Omega_{0} t_{0}$, and $\nu$ is the dimensionless viscosity coefficient. The induction equation is

$$
\frac{\partial \mathbf{A}}{\partial t}=\varepsilon \beta \nabla h-\nabla \Phi+\left(\mathbf{u}-\varepsilon \frac{\nabla \times \mathbf{B}}{n}\right) \times \mathbf{B}-\eta \nabla \times \mathbf{B},
$$

where $\mathbf{A}$ is the vector potential, $\Phi$ is the electrostatic potential, $\varepsilon=c /\left(\omega_{\mathrm{pi}} l_{0}\right)$ is the Hall parameter $\left(l_{0}=v_{A} t_{0}\right.$ being our length unit), and $\eta$ is the dimensionless electric resistivity. The expression for the enthalpy density for an ideal and isothermal gas (i.e., $T_{e}=T_{i}=T=$ const.) is

$$
h_{e, i}=h=\ln n \text {. }
$$

In the corotating reference frame, centered at $r=r_{0}$, we assume a local Cartesian small box (with side size $\Delta \ll r_{0}$ ) such that $x$ is oriented in the radial direction and $y$ is along the azimuthal direction (i.e., $\hat{\mathbf{r}} \rightarrow \hat{\mathbf{x}}$ and $\hat{\phi} \rightarrow \hat{\mathbf{y}}$ ).

Assuming an external magnetic field $B_{0} \hat{\mathbf{z}}$ and an externally applied linear shear flow to a small parcel of plasma located at a radial distance $r_{0}$, the velocity and the magnetic vector fields can be written in terms of the three-dimensional small-scale fields $\mathbf{u}$ and $\mathbf{b}$ as follows:

$$
\mathbf{B}=B_{0} \hat{\mathbf{z}}+\mathbf{b}(z, t) \quad \mathbf{u}=-s x \hat{\mathbf{y}}+\mathbf{u}(z, t),
$$

where

$$
s=-t_{0} \partial_{x} u_{y}
$$

is the externally applied and constant shear. The external magnetic field $B_{0} \hat{\mathbf{z}}$ ( $B_{0}=1$ in the dimensionless version) is distorted by the applied shear flow, developing perpendicular components on both the velocity and the magnetic vector fields.

We adopt the shearing-box approximation (see Hawley et al. 1995 and Umurhan \& Regev 2004 for details on the shearingbox approach). The dimensionless Hall-MHD shearing-box equations are the following:

$$
\begin{aligned}
&\left(\partial_{t}-s x \partial_{y}\right) n+\nabla \cdot(n \mathbf{u})=0 \\
&\left(\partial_{t}-s x \partial_{y}\right) \mathbf{u}+(\mathbf{u} \cdot \nabla) \mathbf{u}=--2 \beta \nabla h+\frac{\nabla \times \mathbf{B} \times \mathbf{B}}{n} \\
&-2 \Omega \hat{\mathbf{z}} \times \mathbf{u}+s u_{x} \hat{\mathbf{y}}+v \nabla^{2} \mathbf{u},
\end{aligned}
$$

$$
\left(\partial_{t}-s x \partial_{y}\right) \mathbf{B}=\nabla \times\left(\mathbf{u}-\varepsilon \frac{\nabla \times \mathbf{B}}{n}\right) \times \mathbf{B}-s B_{x} \hat{\mathbf{y}}+\eta \nabla^{2} \mathbf{B} .
$$

From this set of equations, we analyze the influence of the Hall term in two cases of interest: in Section 5, we study nonrotating $(\Omega=0$ and $s \neq 0)$ plasmas embedded in a large-scale shear flow; in Section 6 we study differentially rotating disks $(\Omega \neq 0$ and $s=a \Omega$ where $a$ comes from a generic profile of differential rotation given by $\left.\Omega(r)=\Omega_{0}\left(r / r_{0}\right)^{-a}\right)$.

\section{DISPERSION RELATION}

After linearizing the Hall-MHD equations with the onedimensional geometric setup described in the previous section by Equation (5), the system splits into two subsystems. One of them corresponds to the longitudinal modes associated to sound waves propagating along the magnetic field, i.e.,

$$
\begin{gathered}
\partial_{t} \delta n=-\partial_{z} u_{z}, \\
\partial_{t} u_{z}=-2 \beta \partial_{z} \delta n,
\end{gathered}
$$

where $\delta n$ are small perturbations from a spatially uniform particle density, i.e., $n=1+\delta n$. The other subsystem corresponds to the perpendicular degrees of freedom described by

$$
\begin{gathered}
\partial_{t} \mathbf{u}_{\perp}=\partial_{z} \mathbf{b}_{\perp}+\left(\begin{array}{cc}
0 & 2 \Omega \\
(s-2 \Omega) & 0
\end{array}\right) \mathbf{u}_{\perp}, \\
\partial_{t} \mathbf{b}_{\perp}=\partial_{z} \mathbf{u}_{\perp}+\left(\begin{array}{cc}
0 & 0 \\
-s & 0
\end{array}\right) \mathbf{b}_{\perp}+\varepsilon\left(\begin{array}{cc}
0 & 1 \\
-1 & 0
\end{array}\right) \partial_{z z}^{2} \mathbf{b}_{\perp} .
\end{gathered}
$$

Equations (10)-(13) show that the perpendicular part $\left(\mathbf{u}_{\perp}, \mathbf{b}_{\perp}\right)$ of the linear dynamics (i.e., Equations (12) and (13)) remain fully decoupled from the longitudinal part $\left(\delta n, u_{z}\right)$ (i.e., Equations (10) and (11)), which is responsible for the propagation of acoustic waves.

Assuming that the components of the perpendicular modes $\mathbf{u}_{\perp}$ and $\mathbf{b}_{\perp}$ are proportional to $e^{i(k z-\omega t)}$, this set of equations leads to the following dispersion relation:

$$
\omega^{4}-2 C_{2} \omega^{2}+C_{0}=0
$$

where

$$
\begin{gathered}
C_{2}(k)=\frac{\varepsilon^{2}}{2} k^{4}+\left(1-\frac{\varepsilon s}{2}\right) k^{2}+\Omega(2 \Omega-s), \\
C_{0}(k)=k^{2}[1+\varepsilon(2 \Omega-s)]\left[k^{2}(1+2 \varepsilon \Omega)-2 s \Omega\right] .
\end{gathered}
$$



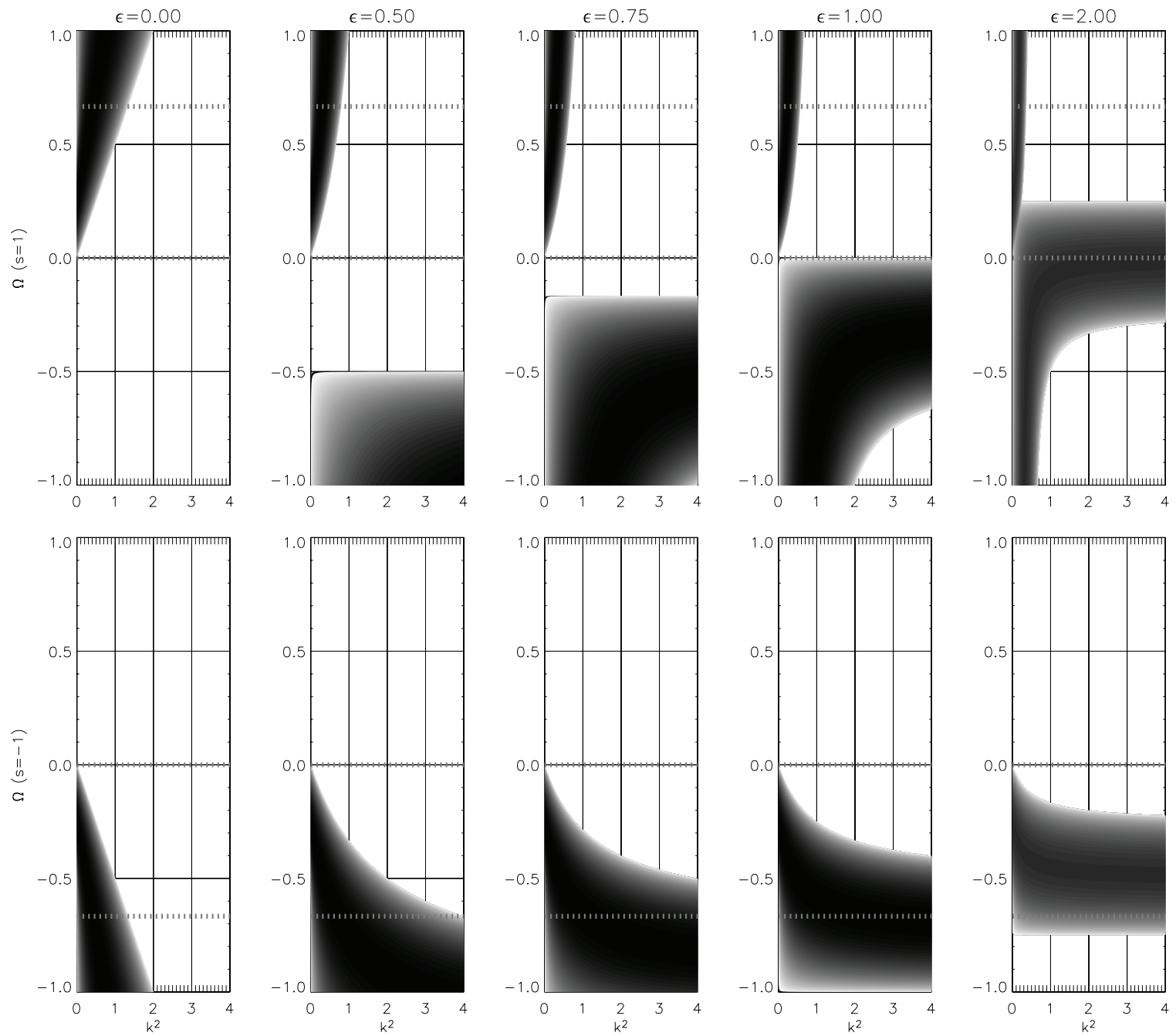

Figure 1. Contour levels of the growth rate for different Hall parameter values on the $\left(\Omega, k^{2}\right)$ plane for positive $(s=+1$, top panel) and negative shear $(s=-1$, bottom panel). Stable regions are white and darker shades correspond to larger instability growth rates. The Keplerian $(\Omega=s / a)$ and non-rotational $(\Omega=0)$ cases are shown by the horizontal dotted gray lines.

Note that this particular dispersion relation is a bi-quadratic polynomial with coefficients depending on $k^{2}$. Therefore, the solutions of the dispersion relation can be written as

$$
\omega_{ \pm}^{2}=C_{2} \pm \sqrt{C_{2}^{2}-C_{0}} .
$$

The set of relevant parameters arising in the linearized HallMHD equations (Equations (7)-(9)) are: the mode wavenumbers (the problem depends on $k^{2}$ ), the Hall parameter $\varepsilon$, the rotation frequency $\Omega$, the local shear $s$ which is the $z$-component of the vorticity (i.e., $\mathbf{w}=\nabla \times \mathbf{u}=(0,0, s)$ ), and the external magnetic field in units of the Alfvénic velocity $v_{A}$. The rotation frequency, the local vorticity, and the external magnetic field are actually vector quantities $(\boldsymbol{\Omega}, \mathbf{w}$, and $\mathbf{B})$, which in this one-dimensional version mean that they can only be aligned or anti-aligned to one another. If we choose the magnetic field along $\hat{\mathbf{z}}$, there are four possible cases, depending on the orientations of the local vorticity $\mathbf{w}$ and the rotation frequency $\boldsymbol{\Omega}$. Up until now, the time unit $t_{0}$ remained undetermined. Therefore, we choose $t_{0}$ so that $\left|\partial_{x} u_{y}\right|=1$, and therefore $s= \pm 1$. We explore the branches $\omega_{+}^{2}$ and $\omega_{-}^{2}$ in the space of parameters given by $\varepsilon, \Omega$, and $k^{2}$. We find that only $\omega_{-}^{2}$ can lead to an instability whenever $\omega_{-}^{2}<0$.
In Figure 1, we show a sequence of plots of the growth rate $\gamma_{-}\left(\right.$with $\left.\gamma_{-}^{2}=-\omega_{-}^{2}\right)$ in the parameter plane $\left(\Omega, k^{2}\right)$ for several values of the Hall parameter. The upper panels of Figure 1 correspond to $s=1$ and the lower panels correspond to $s=-1$. Note that $\Omega$ can be either positive or negative. The horizontal dotted gray lines represent the two cases of interest which we analyze in the following sections: non-rotating flows $(\Omega=0$ and $s \neq 0)$ and differentially rotating Keplerian flows $(\Omega \neq 0$ and $s=a \Omega$ where $\mathrm{a}=3 / 2$; in this set of units $\Omega=s / a= \pm 2 / 3)$. As shown in Figure 1, if the shear is aligned with the magnetic field (positive shear), the non-rotating case becomes unstable for $\varepsilon>1$. In particular, all wavenumbers are unstable for $s=1$ and $\varepsilon>0$, showing an asymptotic behavior for $k^{2} \rightarrow \infty$. On the other hand, if the shear is anti-aligned $(s=-1)$, there are no unstable modes for the non-rotating case. Meanwhile the Keplerian case always has unstable wavenumbers, but they are modified by the different values of the Hall parameter. Figure 1 clearly shows that the $s>0$ and $s<0$ cases are entirely different, except for the purely MHD case $(\varepsilon=0)$. In all cases considered, there is an interval of rotation frequencies for which all wavenumbers are unstable. In the cases with positive shear $(s=1)$, the unstable strip is given by $-1 / 2 \varepsilon<\Omega<(\varepsilon-1) / 2 \varepsilon$. 


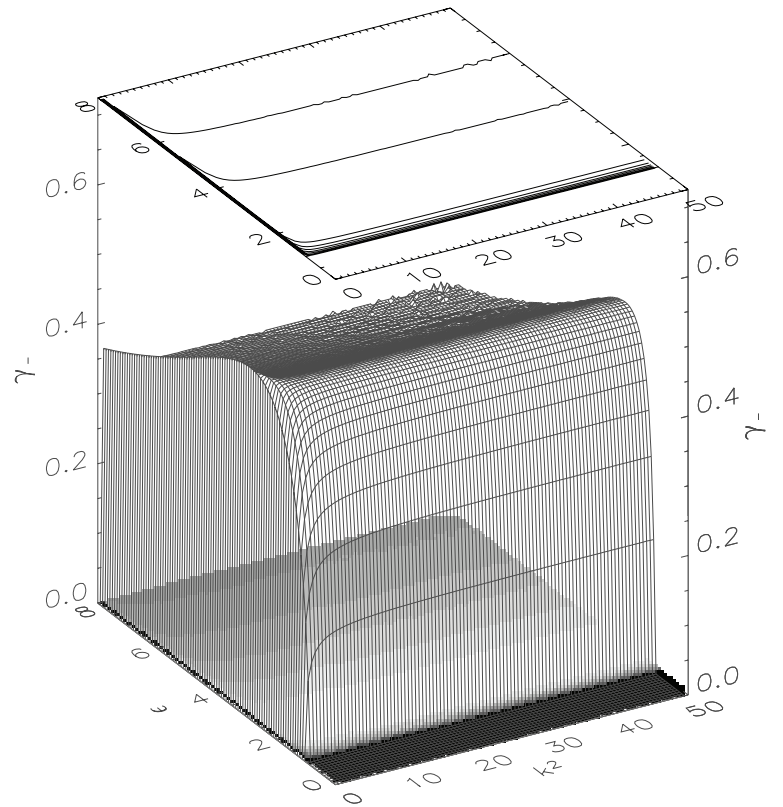

Figure 2. Growth rate of the unstable branch as a function of wavenumber and Hall parameter (i.e., $\left.\gamma_{-}\left(k^{2}, \varepsilon\right)\right)$ for a shear flow $(s=1)$ without rotation. A contour plot is overlaid at the top and a gray-scale image is shown at the bottom.

For the cases with negative shear $(s=-1)$, it is given by $-(\varepsilon-1) / 2 \varepsilon \leqslant \Omega \leqslant-1 / 2 \varepsilon$.

\section{NUMERICAL SIMULATIONS}

A natural first step is to test the growth rates arising from the dispersion relation with the numerical results from simulations. We have explored two different cases: non-rotating shear flows in Section 5 and differentially rotating flows (which can locally be approximated by a linear shear flow) in Section 6. We performed hydromagnetic compressible simulations in one dimension using the PENCIL CoDE ${ }^{5}$ (Brandenburg \& Dobler 2002), a high-order finite-difference numerical suite for compressible MHD flows.

The simulations have been done with externally imposed shear, either with or without rotation. For the initial condition, we assume a monochromatic Alfvén wave traveling in $\hat{\mathbf{z}}$ with a very small amplitude. The resistivity and viscosity coefficients (i.e., $\eta$ and $v$ ) were small enough to be non-negligible only at the smallest spatial scales. The computational domain is $2 \pi$ periodic in the $\hat{\mathbf{z}}$ direction, which is both the rotation axis and the orientation of the external magnetic field.

\section{HALL MAGNETO-SHEAR INSTABILITY (HALL-MSI)}

From Equations (15) and (16), we derive the corresponding coefficients for the case of non-rotating plasmas (i.e., $\Omega_{0}=0$ ) embedded in large-scale shear flows, i.e.,

$$
\begin{gathered}
C_{2}=\frac{\varepsilon^{2} k^{4}}{2}+\left(1-\frac{\varepsilon}{2}\right) k^{2}, \\
C_{0}=k^{4}(1-\varepsilon) .
\end{gathered}
$$

Note that Equations (15) and (16) for the particular case $\Omega=0$ depend on the combination $\varepsilon s$, with $s= \pm 1$ (depending

\footnotetext{
5 http://code.google.com/p/pencil-code
}

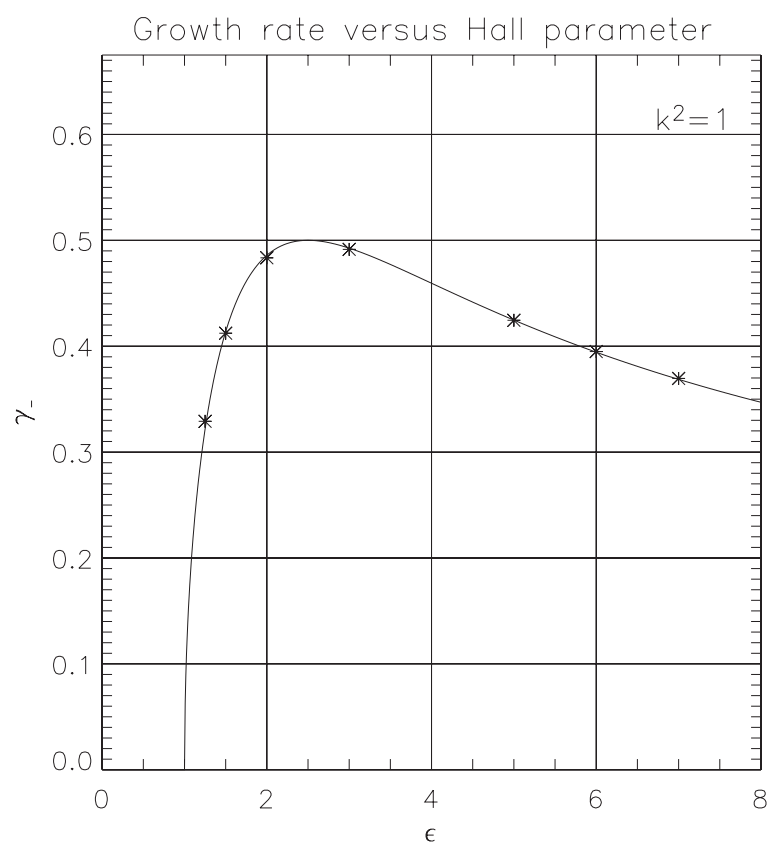

Figure 3. Overlap between the theoretical model (solid line) and the simulations (asterisk) for $k=1$ for a shear flow $(s=1)$ without rotation.

on the relative orientation of the shear's vorticity with respect to the external magnetic field). In Equations (18) and (19) we simply replaced $\varepsilon s$ with a "signed $\varepsilon$." Positive values of $\varepsilon$ correspond to a shear-related vorticity $\mathbf{w}$ aligned with the external magnetic field, while negative values of $\varepsilon$ describe anti-aligned configurations.

These coefficients are now considerably simpler and allow a straightforward study of this instability. Note that the even simpler case of $\varepsilon=0$ is stable, since $C_{2}=k^{2}$ and $C_{0}=k^{4}$, which therefore describes the propagation of Alfvén waves along the $\hat{\mathbf{z}}$ direction. Therefore, when the Hall current is neglected, an external shear flow is unable to drive an instability, as expected. On the other hand, it is straightforward to prove that $C_{2}^{2}-C_{0} \geqslant 0$. Consequently, $\omega_{ \pm}^{2}$ are real numbers (see Equation (17)) and there are a priori four possible cases depending on the signs of $C_{0}$ and $C_{2}$. However, there is no region on the $\left(k^{2}, \varepsilon\right)$ plane where $C_{0}>0$ and $C_{2}<0$ simultaneously. Therefore, the condition for instability reduces to $C_{0}<0$, which in turn implies $\varepsilon>1$. Considering the units used for the present study, $\varepsilon>1$ corresponds to $\left|\partial_{x} u_{y}\right|>\omega_{\mathrm{ci}}$ (where $\omega_{\mathrm{ci}}=e B_{0} /\left(m_{i} c\right)$ is the ion-cyclotron frequency), which corresponds to a rather steep linear shear flow, except perhaps for considerably mild external magnetic fields.

In Figure 2, we show the growth rate for the unstable branch $\gamma_{-}\left(k^{2}, \varepsilon\right)$. The instability region extends all the way from $\varepsilon=1$ to $\varepsilon \rightarrow \infty$ and, more importantly, it is unstable for all wavenumbers. At large wavenumbers, the growth rate reaches its maximum value $\gamma_{-}^{\max }=0.5$ at $\varepsilon=2$ (i.e., $\left|\partial_{x} u_{y}\right|>2 \omega_{\mathrm{ci}}$ ). The contour levels of the growth rate $\gamma_{-}$on the $\left(k^{2}, \varepsilon\right)$ diagram, which are overlaid at the top, clearly show an asymptotic behavior toward $k \rightarrow \infty$. This implies that all Fourier modes are unstable for a specific range of values of the Hall parameter.

In Figure 3, a transverse cut of $\gamma_{-}$versus $\varepsilon$ is shown for $k^{2}=1$. There is also a very good agreement between the analytical result (solid line) and numerical results (asterisk) obtained from simulations. The instability region corresponds to positive values of $\varepsilon$ which implies that $\mathbf{S}$ and $\mathbf{B}$ are aligned. 


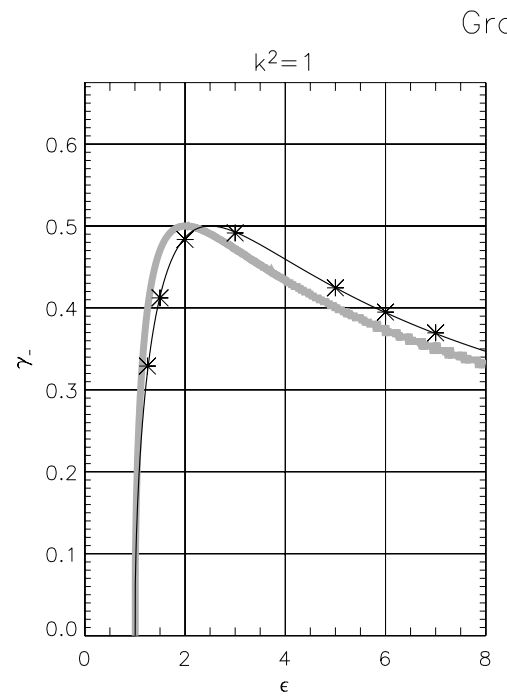

Growth rate versus Hall parameter
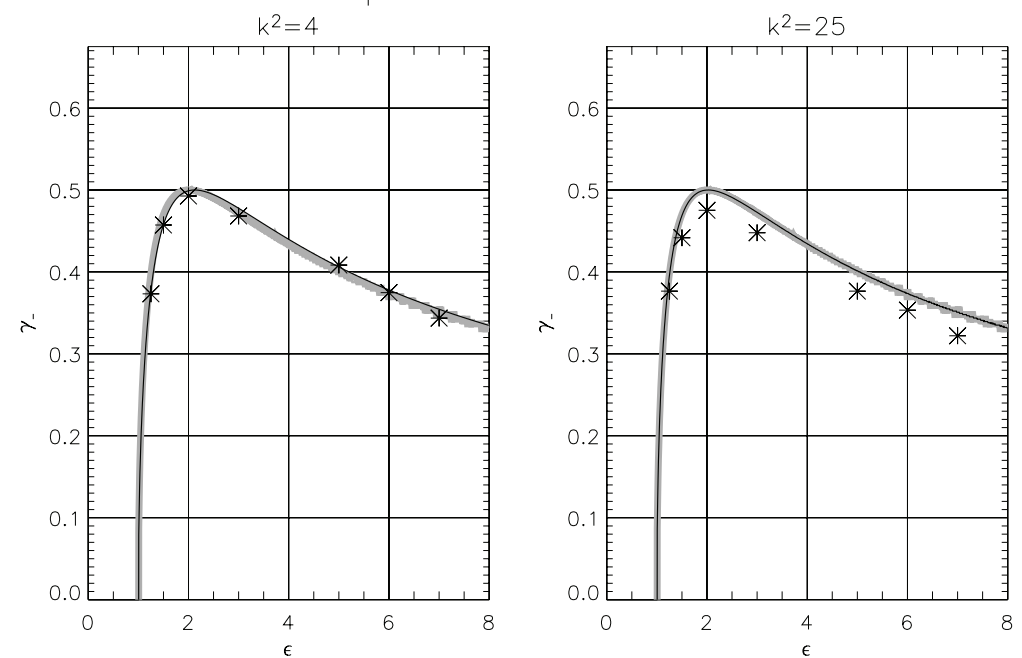

Figure 4. Overlap between the theoretical model (solid line) and the simulations (asterisk) for $k=1, k=2$, and $k=5$. The asymptotic behavior for $k^{2} \rightarrow \infty$ (gray line) is indicated in the three cases.

In Figure 4, we show profiles of the growth rate as a function of the Hall parameter for three different wavenumbers $(k=1$, $k=2$, and $k=5$ ) in order to display the asymptotic behavior when $k^{2} \rightarrow \infty$, represented by a gray curve in each panel. Note that in the asymptotic limit, the frequency of the unstable mode becomes gradually independent of $k$. This causes the convective and Hall terms on Equation (13) (i.e., the first and third terms on the right-hand side) to grow as $k^{2}$, while the remaining terms become comparatively negligible. Therefore, the large$k$ asymptotic regime is in fact approximately independent of $k$.

Although we did not explore it in this paper, this instability also extends toward small wavenumbers (i.e., $k \ll 1$ in our units). The maximum growth rate $\gamma_{-}^{\max }=0.5$ is actually attained for all parameter values $\varepsilon$ and $k$ satisfying $(\varepsilon-2) k^{2}=1 / 2$. This particular regime might correspond to the one considered by Rüdiger \& Kitchatinov (2005) for very large values of $\varepsilon$, which they termed as "shear-Hall." However, it must be pointed out that their study was performed for differentially rotating plasmas. In the asymptotic limit of very large $\varepsilon$ while $\varepsilon k^{2}$ remains constant, the effect of rotation becomes gradually unimportant and the instability is driven by the velocity shear associated with the differentially rotating flow. To the best of our knowledge, the Hall-MSI (as a process driven by a mechanism unrelated with a differential rotation profile) was only explored in its linear regime by Kunz (2008) for weakly ionized plasmas in a three-dimensional geometry.

\section{HALL MAGNETO-ROTATIONAL INSTABILITY (HALL-MRI)}

For the large-scale behavior of accretion disks many results have been obtained within the framework of MHD. If the accretion disk displays differential rotation in the presence of magnetic fields, the MRI appears as the most promising candidate (Velikhov 1959; Chandrasekhar 1961; Balbus \& Hawley 1991). The MRI has been broadly tested by three-dimensional numerical simulations which show that the strong turbulence generates and enhances angular momentum transport efficiently (Brandenburg et al. 1995; Hawley et al. 1995; Matsumoto \& Tajima 1995). However, kinetic plasma phenomena such as the Hall effect, might play an important role in the development of small-scale instabilities and turbulence. In particular, the influence of the Hall currents on the MRI has been considered in the linear (Wardle 1999; Balbus \& Terquem 2001; Rüdiger \& Kitchatinov 2005; Devlen \& Pekünlü 2007) as well as in the nonlinear regimes (Sano \& Stone 2002a, 2002b).

As shown in the previous section, in the presence of differential rotation the constant shear becomes $s=a \Omega=a \Omega_{0} t_{0}$. In MRI studies it is customary to use $t_{0}=1 / \Omega_{0}$, which in turn implies that $s=a$ (see, for instance, Balbus \& Hawley 1991). We adopt these units in the present section, so that our results can be compared with existing results in MRI in a straightforward manner. The characteristic length scale is therefore $l_{0}=v_{A} / \Omega_{0}$. In the presence of differential rotation, the coefficients of the dispersion relation are

$$
\begin{gathered}
C_{2}=\frac{\varepsilon^{2} k^{4}}{2}+\left(1-\frac{\varepsilon a}{2}\right) k^{2}+(2-a), \\
C_{0}=k^{2}[1+\varepsilon(2-a)]\left[k^{2}(1+2 \varepsilon)-2 a\right] .
\end{gathered}
$$

Note that the cases where the rotation frequency $\boldsymbol{\Omega}$ is antialigned with the external magnetic field (i.e., $\Omega$ in Equations (15) and (16)) can be described in Equations (20) and (21) with negative values of the Hall parameter $\varepsilon$. Since Equations (15) and (16) for $\Omega \neq 0$ and $s=a \Omega$ depend on the combination $\varepsilon \Omega$, with $\Omega= \pm 1$ (depending on the relative orientation of the rotation frequency with respect to the external magnetic field), we can also consider $\varepsilon \Omega$ as a "signed $\varepsilon$." In Hall-MRI, positive (negative) values of $\varepsilon$ correspond to $\Omega$ aligned (anti-aligned) to the external magnetic field.

We study three different rotation profiles: Keplerian $(s=$ $1.5)$, slightly sub-Keplerian $(s=1.25)$, and slightly superKeplerian $(s=1.75)$. The growth rate $\gamma_{-}\left(k^{2}, \varepsilon\right)$ is shown in Figure 5 for the Keplerian case (i.e., $a=3 / 2$ ). Contour levels of this function are also overlaid at the top as well as a gray-scale image is shown at the bottom.

In Figure 6, we present the corresponding contour plots for the following three cases: sub-Keplerian, Keplerian, and superKeplerian. Note that in all of them, $\gamma_{-}$reaches an asymptotic value which is positive and independent of $k^{2}$. Also, the whole unstable region becomes appreciably larger when differential rotation goes from sub-Keplerian to super-Keplerian regimes. 


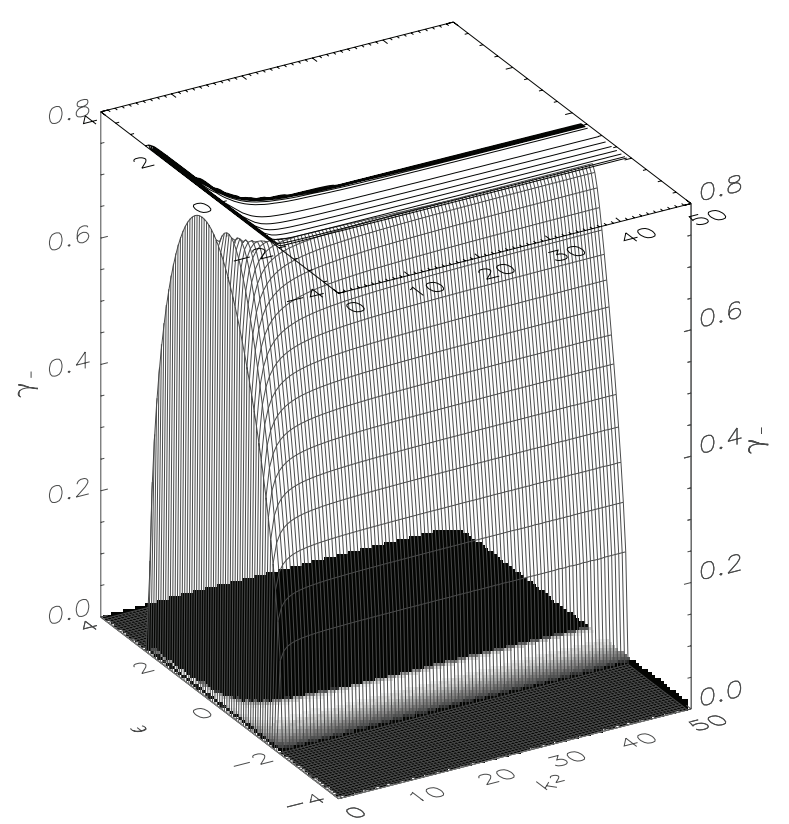

Figure 5. Growth rate of the unstable branch as a function of wavenumber and Hall parameter (i.e., $\gamma_{-}\left(k^{2}, \varepsilon\right)$ ) for a Keplerian rotation profile. A contour plot is overlaid at the top and a gray-scale image is shown at the bottom.

An interesting feature of the Hall effect is the fact that it breaks the MHD symmetry $\mathbf{B} \leftrightarrow-\mathbf{B}$. A direct consequence of this symmetry breaking is a strong change of the instability region in the space of parameters depending on the relative alignment of the external magnetic field and the angular velocity of the disk. Also, the Hall effect qualitatively changes the criterion leading to instability even in cases where the angular velocity increases outward (Balbus \& Terquem 2001; Sano \& Stone 2002a, 2002b).

The instability region can be approximately described through a range of negative values of $\varepsilon$, therefore corresponding to disks where the angular velocity and magnetic field vectors are anti-aligned. For the cases where these two vectors are aligned (i.e., $\varepsilon>0$ ), instability only arises in a narrow region at the very smallest wavenumbers allowed in the shearing box. The instability region in the $\left(\varepsilon, k^{2}\right)$ diagram is confined within two curves of marginal stability, which are determined by the condition $C_{0}=0$, i.e.

$$
\begin{gathered}
\varepsilon=-\frac{1}{2}+\frac{a}{k^{2}}, \\
\varepsilon=-\frac{1}{2-a} .
\end{gathered}
$$

For instance, for Keplerian rotation (i.e., $a=3 / 2$ ) the instability strip at large wavenumbers ranges from $\varepsilon=-1 / 2$ (i.e., Equation (22) for $k \rightarrow \infty$ ) to $\varepsilon=-2$ (see Equation (23)). As we go to super-Keplerian regimes, Equation (23) indicates that the instability strip widens up considerably, especially when the rotation parameter $a \rightarrow 2$.

The particular case of the standard MRI corresponds to $\varepsilon=0$, for which we recover the classical result (Balbus \& Hawley 1991). The highest growth rate for the unstable branch at $k^{2}=15 / 16$ is $\gamma_{-}^{\max }=0.75$ and the range of unstable wavenumbers is restricted to $0<k^{2}<3$. For the sub-Keplerian and super-Keplerian cases, the highest growth rates at the same $k^{2}$ are, respectively, 0.625 and 0.875 , although each maximum is achieved for different values of the Hall parameter. Also, the maximum growth rate can be obtained from the calculation of the local Oort A value of the rotation profile (see, for instance, Balbus \& Terquem 2001).

As mentioned at the beginning of this section, the influence of the Hall effect in the context of accretion disks has been previously analyzed. For instance, we obtain the same results for $k \rightarrow \infty$ reported by Wardle (1999), Balbus \& Terquem (2001), and Devlen \& Pekünlü (2007). In particular, Figures 5 and 6 can be straightforwardly compared with Figures 1 and 5 of Balbus \& Terquem (2001). Also, the dispersion relation presented by Rüdiger \& Kitchatinov (2005) in their Equation (7), in the ideal limit, corresponds to the coefficients shown in Equations (20) and (21).

In Figure 7 , we display transverse cuts of $\gamma_{-}$versus $\varepsilon$ for $k^{2}=1$. The three rotation profiles (i.e., sub-Keplerian (dotted line), Keplerian (solid line), and super-Keplerian (dashed line)) are overlaid for comparison. As we go to super-Keplerian profiles, the instability range of values of $\varepsilon$ is broadened and the growth rates become progressively larger. We performed several

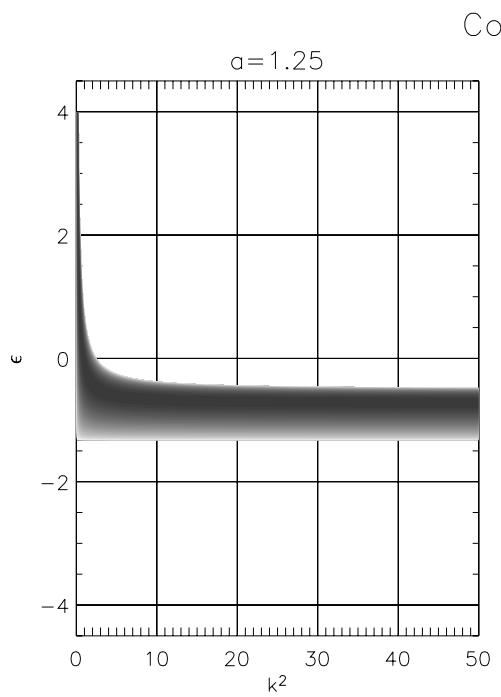

Contour levels of the growth rate
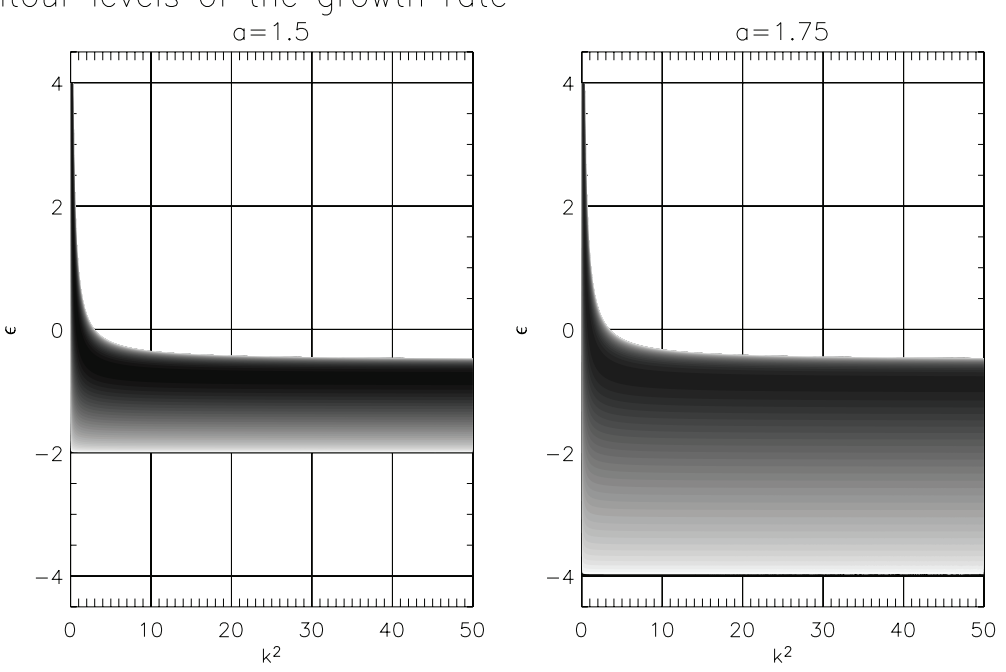

Figure 6. Contour levels of the instability growth rate $\gamma_{-}$on the $\left(k^{2}, \varepsilon\right)$ plane for sub-Keplerian $(s=1.25)$, Keplerian $(s=1.5)$, and super-Keplerian $(s=1.75)$ cases. Stable regions are white and darker shades correspond to larger instability growth rates. 


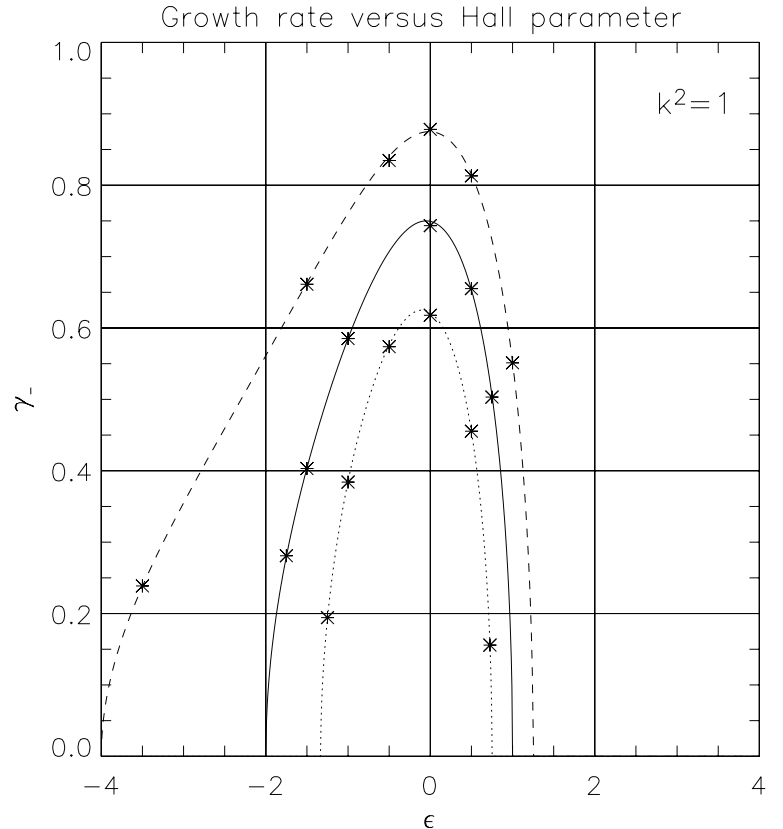

Figure 7. Overlap between the theoretical model and the simulations (asterisk) for $k=1$ and for three differential rotation profiles: sub-Keplerian (dotted line), Keplerian (solid line), and super-Keplerian (dashed line). The well-known MRI corresponds to $\varepsilon=0$ where the highest growth rate for the unstable branch is achieved.

numerical simulations for different values of $\varepsilon$ and $a$, and the corresponding results are indicated with asterisks, showing a very good agreement with the analytical results.

In Figure 8, we show three different cuts of $\gamma_{-}$versus $\varepsilon$ corresponding to three different values of wavenumbers for the Keplerian flow. Figure 8 exhibits a good correspondence between the numerical simulations and the analytical results. Note that the asymptotic behavior (i.e., for $k \rightarrow \infty$ ) indicated with gray trace is gradually approached as the wavenumber increases. Just as for the Hall-MSI case in Section 5, the frequency of the unstable mode becomes gradually independent of $k$. In fact, the approximate balance between the convective and Hall terms in Equation (13) is such that the large- $k$ asymptotic regime becomes independent of $k$.
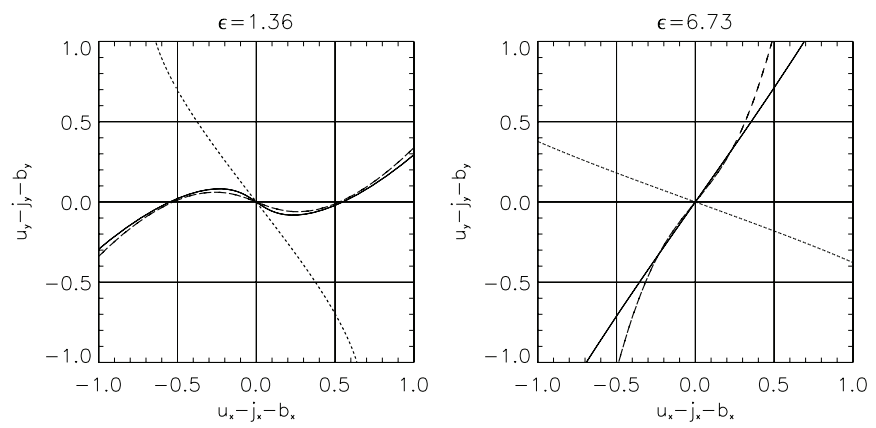

Figure 9. Parametric representation of proton velocity (solid line), current density (dashed line), and magnetic field (dotted line) for Hall-MSI and for different values of the Hall parameter (labeled).

The sub/super-Keplerian profiles can be relevant in different astrophysical contexts. There are phenomenological constraints imposed on compact object (in particular, neutron stars and black holes) properties such as masses, spins, and sizes. The emitting regions of accretion disks are constrained by the assumption that super-Keplerian oscillation frequencies cannot be observed from any radius. Therefore, the frequency of the lowest order linear hydrodynamic modes has to be smaller than the local Keplerian value. The validity of this assumption is investigated by Mao et al. (2009). In Kato et al. (2009) the evolution of the MRI is studied for weakly ionized protoplanetary disks, assuming a radially non-uniform magnetic field. For this physical configuration, the authors find that a zone with a super-Keplerian velocity emerges as a result of the non-uniformly growing MRI turbulence. Likewise, in Kato et al. (2010), the dust particles pile up at the boundaries of sub/super-Keplerian regions, in such a way that the dust density becomes large enough for the subsequent gravitational instability to set in. This result suggests a possible route to planetesimal formation from the dust particle in a protoplanetary disk via the non-uniformly excited MRI. In accretion processes for which infalling matter does not really fall onto the disk surface, but onto the disk outer edge (which means that the disk accretes from the side instead of from the top), it may be necessary to consider deviations from Keplerian rotation profile. In this context, the problem of sub-Keplerian accretion disks requires particular attention (Chakrabarti 1995;
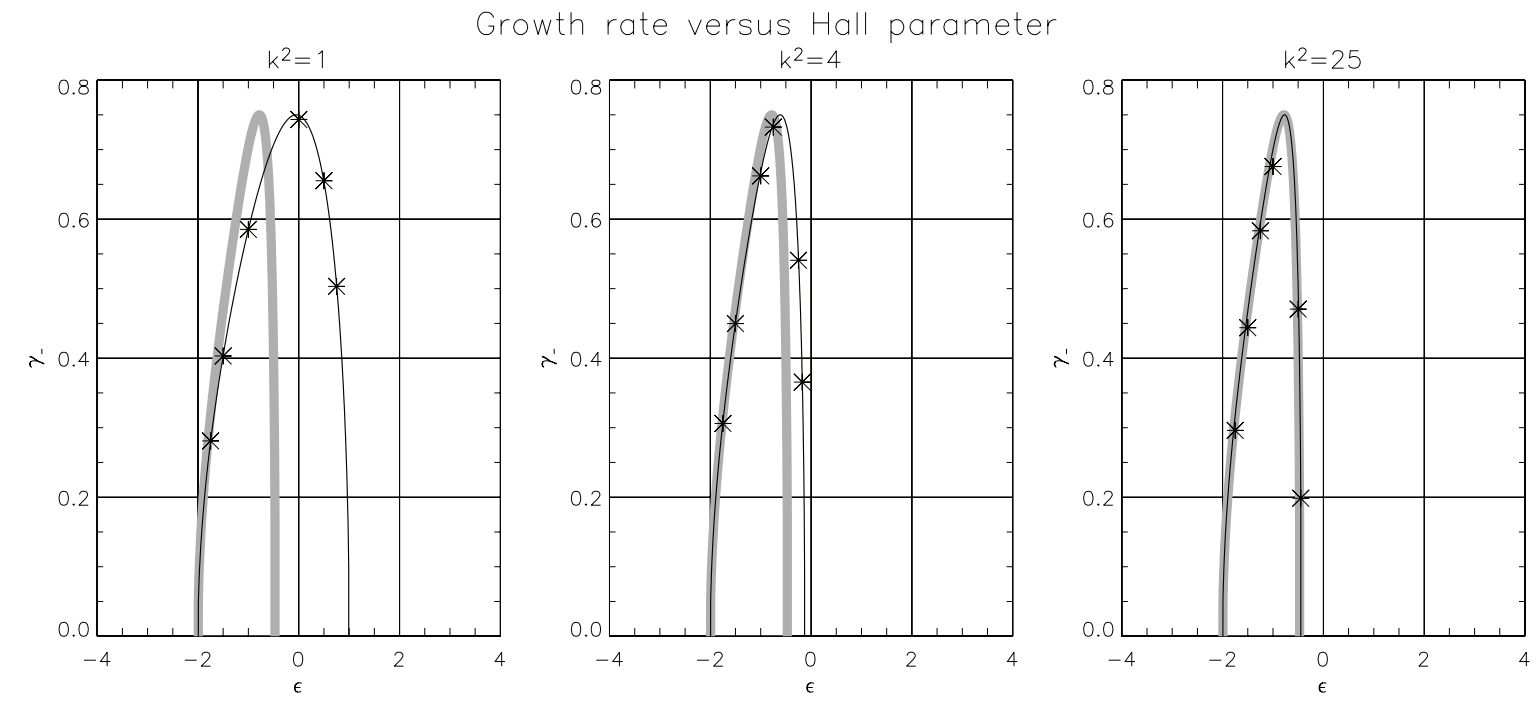

Figure 8. Overlap between the theoretical model (solid line) and the simulations (asterisk) for $k=1, k=2$, and $k=5$ for Keplerian rotation profile. The asymptotic behavior for $k^{2} \rightarrow \infty$ (gray line) is indicated in the three cases. 

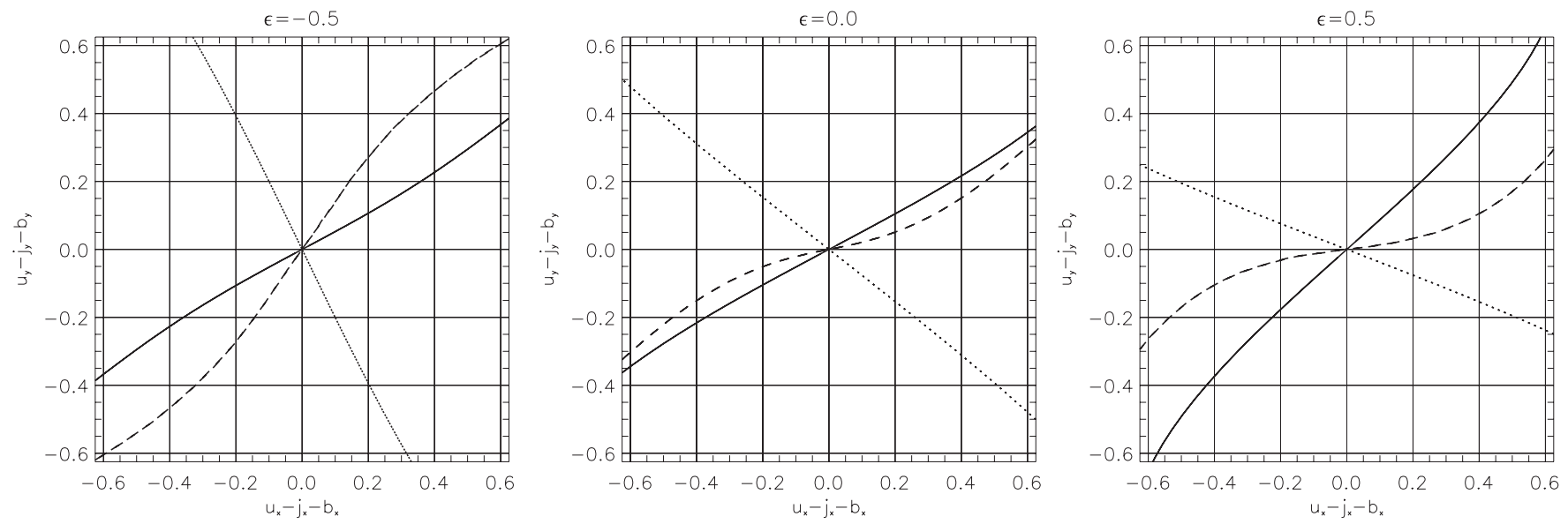

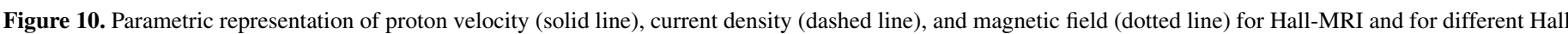
regimes: negative $(\varepsilon=-0.5)$, MHD $(\varepsilon=0)$, and positive $(\varepsilon=0.5)$.
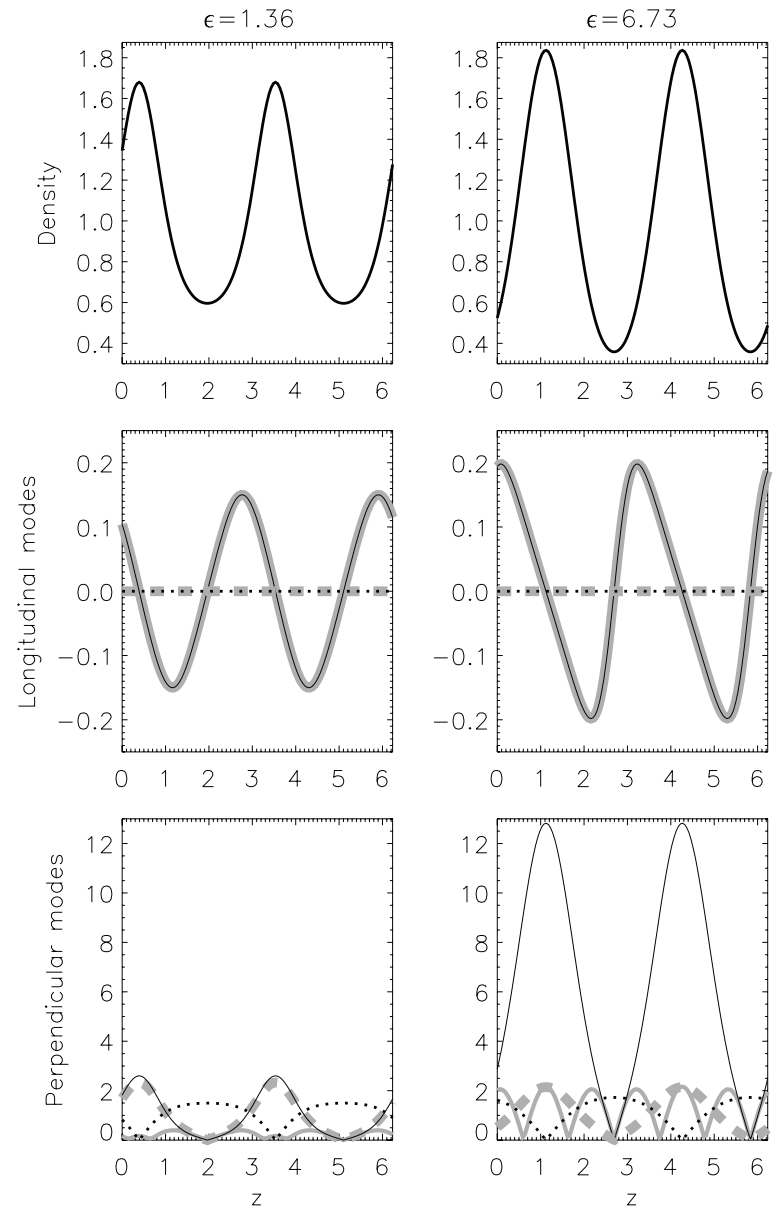

Figure 11. Nonlinear behavior of Hall-MSI for $\beta=1$ and for two different values of the Hall parameter (labeled). The upper panels show the particle density, the central panels display the longitudinal modes (i.e., the $z$-components), and the lower panels show the perpendicular modes (i.e., $(x, y)$-components). There are four vector fields represented: proton (solid black line) and electron (solid gray line) velocities, current density (dashed gray line), and the magnetic fields (dotted black line).

Hueso \& Guillot 2005; Visser \& Dullemond 2010). A strong magnetization can make the disk surrounding young stellar objects rotate at sub-Keplerian rate (see, for instance, Shu et al. 2008 and Paardekooper 2009).

\section{NONLINEAR BEHAVIOR}

In the nonlinear regime, the Hall-MHD equations with the geometric configuration depicted by Equation (5) are as follows:

$$
\begin{gathered}
\partial_{t} \delta n=-\partial_{z} u_{z}-\partial_{z}\left(u_{z} \delta n\right), \\
\left(\partial_{t}+u_{z} \partial_{z}\right) u_{z}=-2 \beta \partial_{z} \delta n-\frac{1}{1+\delta n} \partial_{z}\left(\frac{b_{\perp}^{2}}{2}\right) \\
\left(\partial_{t}+u_{z} \partial_{z}\right) u_{\perp}=\frac{1}{1+\delta n}\left(1+b_{z}\right) \partial_{z} b_{\perp}+\left(\begin{array}{cc}
0 & 2 \Omega \\
(s-2 \Omega) & 0
\end{array}\right) u_{\perp}, \\
\left(\partial_{t}+u_{z} \partial_{z}\right) b_{\perp}=-b_{\perp} \partial_{z} u_{z}+\partial_{z} u_{\perp}+\left(\begin{array}{cc}
0 & 0 \\
-s & 0
\end{array}\right) b_{\perp}+\varepsilon \\
\times\left(\begin{array}{cc}
0 & 1 \\
-1 & 0
\end{array}\right) \partial_{z}\left(\begin{array}{cc}
\left.1+b_{z}\right) \frac{\partial_{z} b_{\perp}}{1+\delta n}
\end{array}\right) .
\end{gathered}
$$

These nonlinear equations show the interplay between the longitudinal (acoustic waves) and perpendicular modes (Hall-MSI or Hall-MRI). Even if the acoustic modes are initially turned off, the nonlinear modes coupling with the perpendicular modes will eventually turn them on.

We analyze two different regimes according to the value of the plasma parameter: $\beta \cong 1$ and $\beta \gg 1$ (high temperature). In the astrophysical context of accretion disks, the MRI instability requires that the unstable wavelengths are smaller than the disk thickness, thus the large $\beta$ regimes are therefore more relevant. However, we numerically verify that the nonlinear regime for the very large beta is associated with the MHD configuration (i.e., without the Hall parameter).

In Section 3, the dispersion relation determined by Equations (15) and (16) shows that the linear behavior is independent of the plasma parameter, therefore the instability growth rate is the same for both regimes because it is only a function of the wavenumber and the Hall parameter. The root mean square $\left(f^{\mathrm{rms}}=\left\langle\mathbf{f}^{2}\right\rangle^{1 / 2}\right)$ of the proton velocity and the magnetic field present very similar behavior for all cases, with different values of $\varepsilon$ and $\beta$ parameters. It should be noted that, if the growth rate increases, the duration of the linear period decreases, as expected. 

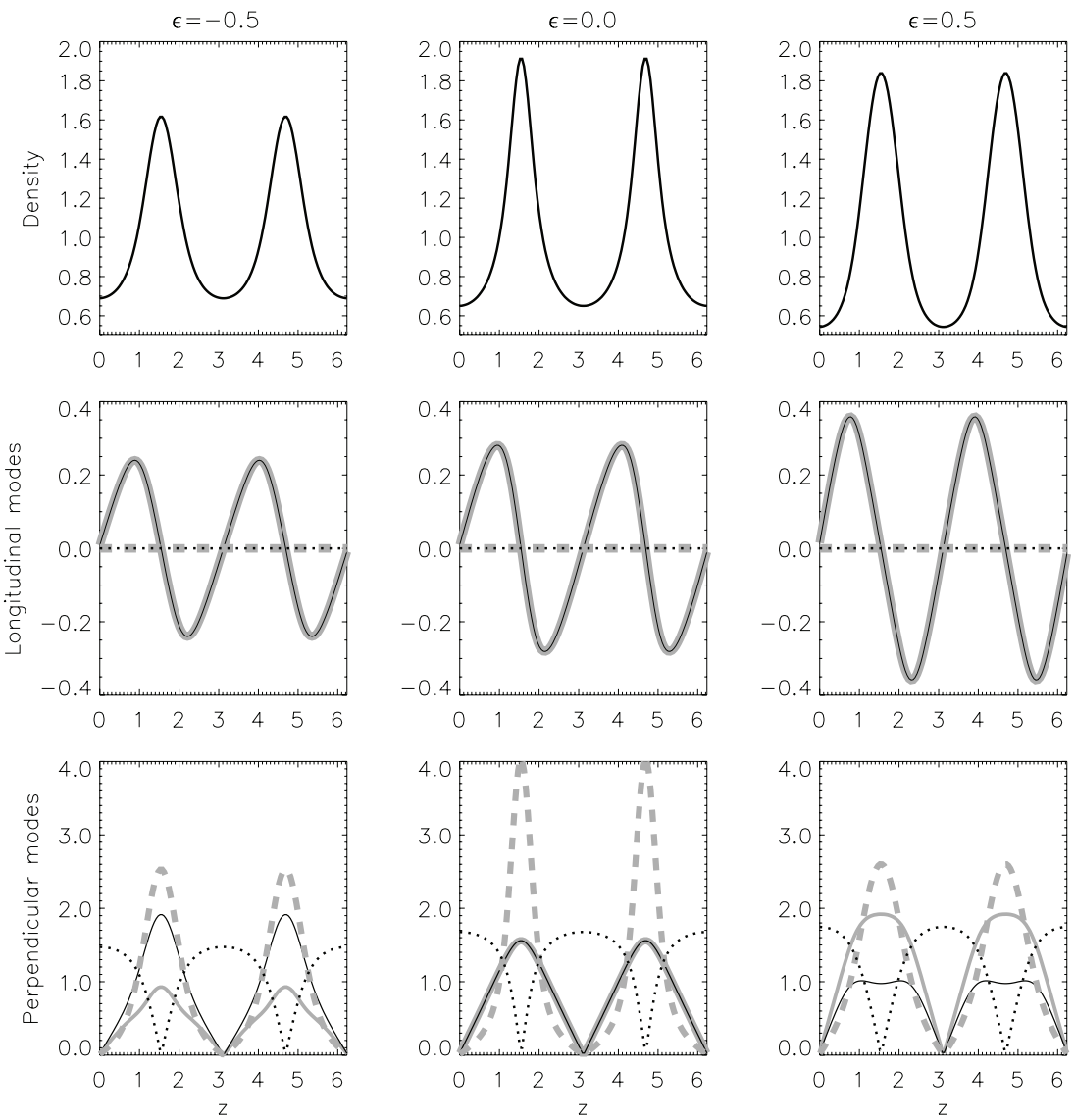

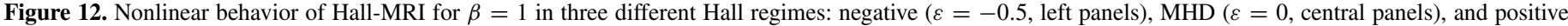

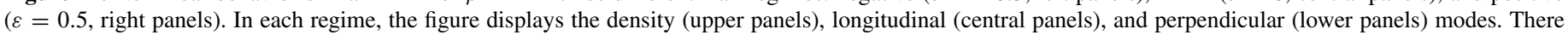

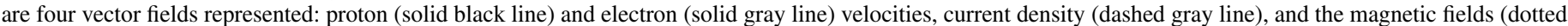
black line).

From Equations (12) and (13), the eigenvectors for the linear regime of the two instabilities (i.e., Hall-MSI and Hall-MRI) can be calculated. For Hall-MSI we obtain

$$
\begin{gathered}
\mathbf{u}_{\perp}=\left(1, \frac{\varepsilon-\left(1+\gamma_{-}^{2}\right)}{\gamma_{-} \varepsilon}\right) u_{x}, \\
\mathbf{J}_{\perp}=\left(\frac{\left(1+\gamma_{-}^{2}\right)}{\varepsilon}, \gamma_{-}\right) u_{x} .
\end{gathered}
$$

Also, it is possible to verify that

$$
\tan \phi_{u}=\frac{\varepsilon-\left(1+\gamma_{-}^{2}\right)}{\gamma_{-} \varepsilon}=\tan \phi_{J}=\frac{\gamma_{-} \varepsilon}{1+\gamma_{-}^{2}} .
$$

Therefore, the proton velocity field and the current density are either aligned if the Hall parameter is positive or anti-aligned if the Hall number is negative (keeping in mind that the unstable modes are associated with positive values of the Hall parameter). For Hall-MRI the eigenvectors are

$$
\begin{gathered}
\mathbf{u}_{\perp}=\left(1, \frac{\gamma_{-}\left(\gamma_{-}^{2}+1+\varepsilon(\varepsilon-a)\right)}{2 \gamma_{-}^{2}+2 \varepsilon(\varepsilon-a)+\varepsilon}\right) u_{x}, \\
\mathbf{J}_{\perp}=\left(\frac{\gamma_{-}^{2}\left(\gamma_{-}^{2}+1+\varepsilon(\varepsilon-a)\right)}{2 \gamma_{-}^{2}+2 \varepsilon(\varepsilon-a)+\varepsilon}+\varepsilon-a,\right. \\
\left.\frac{\gamma_{-}^{2}\left(\gamma_{-}^{2}+1+\varepsilon(\varepsilon-a)\right)}{2 \gamma_{-}^{2}+2 \varepsilon(\varepsilon-a)+\varepsilon}-\gamma_{-}\right) \frac{u_{x}}{\gamma_{-}^{2}+\varepsilon(\varepsilon-a)} .
\end{gathered}
$$

For all values of the Hall parameter and any differential rotation profile (represented by $a$ ), we numerically show that the proton velocity field and the current density are always anti-aligned since $\cos \left(\theta_{u, j}\right)=(\mathbf{u} \cdot \mathbf{J}) /|\mathbf{u}||\mathbf{J}|=-1$.

In order to give a qualitative description of the nonlinear regime, we select particular values of the Hall parameter. For Hall-MSI, we choose them in such a way that the growth rates correspond to $75 \%$ of the maximum, therefore we obtain $\varepsilon=1.36$ and $\varepsilon=6.73$. For Hall-MRI, we adopt values of the Hall parameter such that the corresponding growth rates are comparable to $90 \%$ of the maximum which takes place at $\varepsilon=0$; thus we choose $\varepsilon=-0.5$ and $\varepsilon=0.5$.

In Figures 9 and 10, we display parametric plots of proton velocity (solid line), current density (dashed line), and magnetic field (dotted line) at times corresponding to the nonlinear regime and for different values of the Hall parameter. From Figure 9, which corresponds to Hall-MSI, it seems apparent that, regardless of the values of the Hall parameter, the eigenvectors for the velocity field and the current density are always aligned, thus extending the result shown in Equation (30) to the nonlinear regime as well. Similarly, Figure 10 shows that for Hall-MRI the velocity field and the current density are always anti-aligned as we numerically find in the linear regime.

In both cases, we note that the influence of the nonlinear terms becomes non-negligible whenever the density fluctuations reach about $10 \%$ of the mean value. In particular, the regime becomes strongly nonlinear when the velocity is equal to the Alfvén speed. In the super-Alfvénic regime, for Hall-MSI with a strong 
Hall effect the whole system seems to start reaching a saturation state, and for Hall-MRI with a negative of the Hall parameter the system seems to reach saturation.

Also, the field configurations in the nonlinear stage depend on the value of the Hall parameter. In the Hall-MRI case for $\varepsilon<0$, the $x$-component of the current density is larger than the $y$-component, while for $\varepsilon>0$ the inverse situation occurs. In both cases, the system shows the formation of the current sheet in the larger component. For the MHD case, of course, the electron and proton velocities are the same. In this case, current sheets are present in the $\hat{\mathbf{x}}$ and $\hat{\mathbf{y}}$ directions. In the Hall-MSI case with moderate Hall parameter value, current sheets seem to appear in both directions.

In Figures 11 and 12, the profiles of the density and the vector fields $\mathbf{u}$ (solid black line), $\mathbf{u}_{e}$ (solid gray line), $\mathbf{J}$ (dashed gray line), and $\mathbf{b}$ (dotted black line) are shown in the nonlinear regime as functions of $z$ for Hall-MSI and for Hall-MRI, respectively. Negative (positive) values of the Hall parameter imply that the electron velocity is always smaller (larger) than the proton velocity. A more extreme situation takes place in the Hall-MSI case with the proton velocity very much larger than the electron velocity. In particular, for a strong Hall effect this is clearly shown in Figure 11 (lower panels). In addition, the Hall-MRI case for positive values of the Hall parameter seems to show the formation of two-flow jets. These double jet structures have also been reported by Sano \& Stone (2002a) in two-dimensional simulations. It is relevant to note that the $z$-component of the velocities start to increase when the density fluctuations grow and the nonlinear regime is reached. However, this component of the velocities (for protons and electrons) remains negligible. Figures 11 and 12 also show that the longitudinal modes for protons and electrons are exactly overlapped. Meanwhile, the $z$-component of the magnetic field and the current density are approximately zero.

Moreover, the maximum values of the density are correlated with the maximum of the perpendicular velocities and with the maximum of the current density, i.e., zero magnetic field. Meanwhile, the minimum values of density are in relation with the zeroes of the velocity fields and current density, i.e., maximum magnetic field (see Figures 11 and 12).

Even though the nonlinear regimes discussed in this section show interesting features, many of them might change when we extend our simulations to three dimensions. The purpose of this one-dimensional study was to identify different regimes in the space of parameters of the problem.

\section{CONCLUSIONS}

The present work is a comprehensive study of the instabilities arising from the interplay between the Hall effect and a linear shear flow for a one-dimensional model. In other words, we analyze the role of the Hall effect in shear-driven instabilities. We find that an instability develops when the Hall effect is present, which we term Hall-MSI. Also, we recover the MRI, as a particular case, and we quantitatively evaluate the influence of the Hall currents on it.

More specifically, we investigate the stability of the system in the parameter space set by the wavenumber and the Hall parameter. In non-rotating plasmas, we determine the region in the $\left(k^{2}, \varepsilon\right)$ diagram where the Hall-MSI takes place. In rotating plasmas (such as accretion disks), we examine three cases: subKeplerian, Keplerian, and super-Keplerian. For each rotation profile, we establish the region in the $\left(k^{2}, \varepsilon\right)$ diagram where the Hall-MRI occurs. The standard MRI is recovered in the particular case for zero Hall parameter $(\varepsilon=0)$. In both unstable modes, we find a very good agreement between the theoretical model and the numerical simulations.

In addition, we explore the influence of the plasma parameter in two asymptotic cases: $\beta \cong 1$ and $\beta \gg 1$. We find that the linear behavior is independent of this parameter. In the nonlinear stage of the large beta regimes, the flow dynamics seems to evolve as in an MHD system.

Within the framework of astrophysics, the Hall-MSI could be relevant in the interface between a jet and the surrounding environment where a strong shear is present. Astrophysical jets have a very high degree of collimation, probably as a consequence of the presence of magnetic fields. The onedimensional model adopted in this work, even though quite simple, might properly describe this circumstance: the azimuthal component of the helicoidal velocity field around the jet can be represented as a function of the radial direction and fulfills the periodicity condition in a straightforward fashion. The relevant instability in these strongly sheared flows is Kelvin-Helmholtz, which is a purely hydrodynamic instability. The presence of external magnetic fields modify the corresponding growth rate, depending on their strength and spatial orientation, but typical numbers quoted in the literature remain a small fraction of the imposed velocity shear (see Ferrari 1998, Bodo et al. 1998, and Huba 1994, for results from Hall-MHD simulations). Therefore, the Hall-MSI, with a maximum growth rate 0.5 times the externally imposed shear, is definitely relevant in these strongly sheared flows. On the other hand, it seems clear that a three dimensional extension of the present study is necessary for a more realistic description of this instability, especially when it comes to its nonlinear stage.

This work has been supported by the University of Buenos Aires through grant UBACyT X092/2008 and by the ANPCyT through grant PICT 33370/2005. C.B. is a fellow of CONICET and D.O.G. is a researcher member of CONICET.

\section{REFERENCES}

Balbus, S. A., \& Hawley, J. F. 1991, ApJ, 376, 214

Balbus, S. A., \& Terquem, C. 2001, ApJ, 552, 235

Bodo, G., Rossi, P., Massaglia, S., Ferrari, A., Malagoli, A., \& Rosner, R. 1998, A\&A, 333, 1117

Brandenburg, A., \& Dobler, W. 2002, Comput. Phys. Commun., 147, 471

Brandenburg, A., Nordlund, A., Stein, R. F., \& Torkelsson, U. 1995, ApJ, 446, 741

Brandenburg, A., \& Zweibel, E. G. 1995, ApJ, 448, 734

Chakrabarti, S. K. 1995, Ann. New York Acad. Sci., 759, 546

Chandrasekhar, S. 1961, Hydrodynamic and Hydromagnetic Stability (Oxford: Oxford Univ. Press)

Devlen, E., \& Pekünlü, E. R. 2007, MNRAS, 377, 1245

Ferrari, A. 1998, ARA\&A, 36, 539

Hawley, J. F., Gammie, C. F., \& Stone, J. M. 1995, ApJ, 440, 742

Hollerbach, R., \& Rüdiger, G. 2002, MNRAS, 337, 216

Huba, J. D. 1994, Phys. Rev. Lett., 72, 2033

Hueso, R., \& Guillot, T. 2005, A\&A, 442, 703

Kato, M. T., Fujimoto, M., \& Ida, S. 2010, ApJ, 714, 1155

Kato, M. T., Nakamura, K., Tandokoro, R., Fujimoto, M., \& Ida, S. 2009, ApJ 691, 1697

Kinney, R. M., Chandran, B., Cowley, S., \& McWilliams, J. C. 2000, ApJ, 545 907

Kunz, M. W. 2008, MNRAS, 385, 1494

Mahajan, S. M., Mininni, P. D., \& Gómez, D. O. 2005, ApJ, 619, 1014

Mao, S. A., Psaltis, D., \& Milsom, J. A. 2009, ApJ, 703, 717

Matsumoto, R., \& Tajima, T. 1995, ApJ, 445, 767

Mininni, P. D., Gómez, D. O., \& Mahajan, S. M. 2002, ApJ, 567, L81

Mininni, P. D., Gómez, D. O., \& Mahajan, S. M. 2003a, ApJ, 584, 1120

Mininni, P. D., Gómez, D. O., \& Mahajan, S. M. 2003b, ApJ, 587, 472

Norman, C., \& Heyvaerts, J. 1985, A\&A, 147, 247 
Paardekooper, S.-J. 2009, A\&A, 506, L9

Pandey, B. P., \& Wardle, M. 2008, MNRAS, 385, 2269

Potekhin, A. Y. 1999, A\&A, 351, 787

Rüdiger, G., \& Kitchatinov, L. 2005, A\&A, 434, 629

Rüdiger, G., \& Shalybkov, L. 2004, Phys. Rev. E, 69, 016303

Sano, T., \& Stone, J. M. 2002a, ApJ, 570, 314

Sano, T., \& Stone, J. M. 2002b, ApJ, 577, 534

Shalybkov, D. A., \& Urpin, V. A. 1997, A\&A, 321, 685

Shu, F. H., Lizano, S., Galli, D., Cai, M. J., \& Mohanty, S. 2008, ApJ, 682, L121
Spangler, S. R. 1999, in Proc. Second Guillermo Haro Conf., Interstellar Turbulence, ed. J. Franco \& A. Carraminana (Cambridge: Cambridge Univ. Press), 41

Tajima, T., Cable, S., Shibata, K., \& Kulsrud, R. M. 1992, ApJ, 390, 309

Umurhan, O. M., \& Regev, O. 2004, A\&A, 427, 855

Urpin, V. A., \& Yakovlev, D. 1980, SvA, 24, 303

Velikhov, E. P. 1959, Sov. Phys. JETP, 9, 995

Visser, R., \& Dullemond, C. P. 2010, A\&A, 519, A28

Wardle, M. 1999, MNRAS, 307, 849

Wardle, M., \& Ng, C. 1999, MNRAS, 303, 239 